\title{
计入刚度影响效应的滚动直线导轨几何参数 对润滑性能的影响“
}

\author{
李 栋 刘晓玲 李 磊 郭光福 \\ (青岛理工大学机械与汽车工程学院 青岛 266520)
}

\begin{abstract}
摘要: 为保证滚动直线导轨的精度和振动稳定性, 在装配时通常对导轨副施加预紧载荷。为研究预紧条件下滚动直线导轨的 刚度对其润滑性能的影响, 基于 LG-45 型导轨建立了刚度模型和考虑预紧的润滑分析模型。分析了滚珠直径、沟槽曲率比和 滑块速度对钢-钢和钢- $\mathrm{Si}_{3} \mathrm{~N}_{4}$ 两种接触副系统弹性预紧量的影响, 并讨论了刚度对导轨副润滑性能的影响。结果表明: 在相同 工况下, 与钢-钢接触副相比, 钢 $-\mathrm{Si}_{3} \mathrm{~N}_{4}$ 陶瓷接触副的系统弹性预紧量较小, 系统弹性预紧量受滑块速度影响较大, 而受滚珠 直径和沟槽曲率比的影响较小。滑块速度增大会使得膜厚和压力明显增大; 曲率比增大使得接触区椭圆比减小, 膜厚减小, 油膜压力增大; 滚珠直径增大使得膜厚增大, 油膜压力减小。
\end{abstract}

关键词: 滚动直线导轨; 预紧; 润滑; 刚度; 系统弹性预紧量

中图分类号: TH117

\section{Influence of Geometric Parameters on Lubrication Performance of Rolling Linear Guides Considering Stiffness Effects}

\section{Dong LIU Xiaoling LI Lei GUO Guangfu}

(School of Mechanical and Automotive Engineering, Qingdao University of Technology, Qingdao 266520)

\begin{abstract}
During the assembly of rolling linear guide, preload is necessary of rolling linear guide to improve the machining accuracy and reduce the vibration. In order to study the influence of stiffness of linear rolling guide on its lubrication performance under preload condition, a stiffness model and lubrication analysis model considering preload were established based on LG-45 guide. Effects of ball diameter, groove curvature ratio and speed of the slider on the preloaded amount of the elasticity for steel-steel and steel- $\mathrm{Si}_{3} \mathrm{~N}_{4}$ contact pair system were analyzed. The influence of the stiffness on the lubrication performance was discussed. Results show that, under the same conditions, elastic preload amount of steel-steel contact pair is higher than that of steel-Si ${ }_{3} \mathrm{~N}_{4}$ ceramic contact pair. Effects of ball diameter and groove curvature ratio on the preloaded amountof the system elasticity aresmall, however, theinfluence of the slider velocity on the amount of preload is obvious. The film thickness and pressure will increase obviously with the increase of slider speed. As the curvature ratio increases, the contact ellipse ratio decreases, film thickness decreases, and the film pressure increases. When the diameter of the ball increases, the film thickness increases, but the film pressure decreases.
\end{abstract}

Key words: rolling linear guide; preload; lubrication; stiffness; preload amount of system elasticity

\section{0 前言}

滚动直线导轨是用于精密移动、精准控制、精 准导向的精度相对较高的设备中, 其使用寿命和运

* 国家自然科学基金资助项目(51475250)。20200310 收到初稿, 20201120 收到修改稿
动精度主要由滚珠和导轨之间的摩擦磨损程度决 定。润滑剂可以减少滚珠和导轨之间的摩擦, 从而 提高定位精度和使用寿命。预紧是多数导轨副稳定 运行的前提条件，而导轨的润滑性能又会受到预紧 产生的系统变形量的影响。目前在考虑预紧的导轨 副的刚度分析方面, 学者们做了很多工作。OHTA 和 TANAKA ${ }^{[1]}$ 使用柔性模型对有预紧导轨副的变形 
量进行分析。LI 等 ${ }^{[2]}$ 求解了在预加载荷和外载荷作 用下导轨的法向和切向刚度。张巍等 ${ }^{[3]}$ 通过建立刚 度矩阵, 分析了预紧力、载荷和接触角对导轨刚度 的影响。TONG 等 ${ }^{[4]}$ 提出一个用于计算滚珠直线导 轨刚度矩阵的具有五个自由度载荷和位移的理论分 析模型。RAHMANI 和 BLEICHER ${ }^{[5]}$ 通过对水平托 架上加载的方式来研究不同角度偏转对导轨刚度的 影响。田红亮 ${ }^{[6]}$ 等分析了滚动直线导轨的上部球和 下部球载荷对刚度的影响。DUNAJ 等 ${ }^{[7]}$ 构建了将预 紧载荷等效为刚度这一导轨新模型, 并验证了此模 型。HUNG 等 ${ }^{[8-9]}$ 通过数值模拟和试验研究表明铣床 主轴刀具的动态响应随导轨预紧载荷的变化而变 化。CHING 等 ${ }^{[10]}$ 研究表明, 导轨的预紧越大, 振动 条件下机床主轴头的刚度越高。

上述文献考虑不同因素、不同工况对滚动直线 导轨刚度的影响, 分析了刚度变化对机床性能的 影响。

但上述的研究未考虑由预紧产生的弹性变形对 导轨副润滑性能的影响。在工程实际中, 导轨副的 润滑性能 ${ }^{[18]}$ 与系统的弹性变形有关, 而弹性变形取 决于预紧载荷的大小, 而导轨副常常受预紧载荷的 作用。因此, 需要基于预紧载荷引起的刚度效应, 揭示几何参数对导轨副润滑性能的影响机制, 以指 导导轨副的润滑设计和工程实践。

\section{1 预紧示意图}

对滚动直线导轨施加预紧力后, 取单个滚珠 进行分析, 如图 1 所示, 图 1a 为滑块-滚珠接触 副模型图, $A$ 点为滚珠和顶部滑块滚道的接触点。 图 $1 \mathrm{~b}$ 为滑块-滚珠接触副的预紧等效示意图, 预 紧量为 $h_{00}, w$ 是预紧量 $h_{00}$ 对应的预紧载荷, 预 紧量 $h_{00}$ 为给定值。 $h_{1}$ 是滑块与导轨之间的固定 距离, 二者之间为椭圆接触。滑块以垂直于纸面 的方向运行。

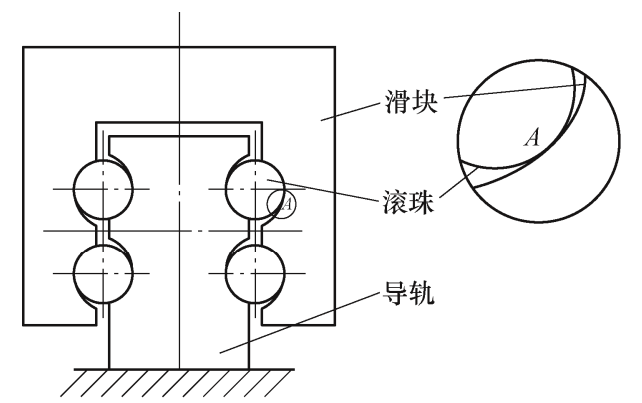

(a) 滑块-滚珠接触副模型

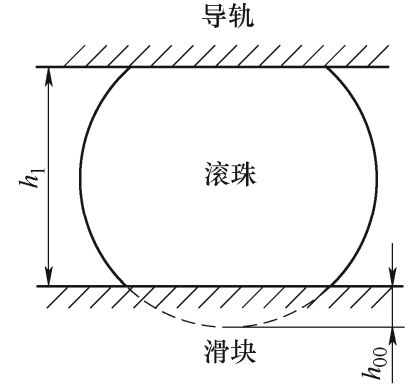

(b) 滑块-流珠接触副的预紧等效示意图

图 1 滑块-滚珠接触副模型

\section{2 预紧后系统刚度的分析}

\section{1 预紧条件下加载后接触角的变化}

为了更简便进行刚度分析，对导轨副作如下简 化假设 ${ }^{[18]}$ 。

(1)预紧前, 导轨初始接触角为 $\theta_{0}$, 预紧后, 导 轨副仅受到垂直方向的载荷作用力 $F_{y}$ 。

(2)弹性变形不超过许用弹性范围。

(3)对导轨副施加垂直载荷后, 产生水平位移 $\delta_{x}$ 和垂直位移 $\delta_{y}$ 后, 导轨的接触角变为 $\theta_{1}$ 。

(4)总变形量 $\delta$ 是由滚珠与导轨接触处的压缩力 $Q$ 决定。

滚珠总变形量 $\delta$ 与导轨的垂直位移 $\delta_{y}$ 的关系见 文献[11]。

$$
\delta_{y} \sin \theta_{1}=\delta\left(1-\frac{1}{\frac{\delta_{y} \tan \theta_{1}}{\delta_{x}}-1}\right)
$$

式中, $\theta_{1}$ 表示对导轨副施加垂直载荷 $F_{y}$ 后的接触角; $\delta_{x} 、 \delta_{y}$ 表示对导轨副施加垂直载荷 $F_{y}$ 后, 导轨副在 水平与垂直方向的位移。

图 $2 \mathrm{a}$ 为预紧前的导轨副几何模型图, 由图可 见, $A 、 B$ 分别表示滑块与固定导轨的曲率中心, $D_{0}$ 是滚珠的参考直径, $r_{\mathrm{a}}$ 和 $r_{\mathrm{b}}$ 分别是滑块和导轨的 曲率半径。 $A B$ 表示导轨曲率中心与滑块的距离, 并 作以下简化: 令 $A B=m_{0}$ 。

$$
A B=r_{\mathrm{a}}+r_{\mathrm{b}}-D_{0}=m_{0}
$$

图 $2 \mathrm{~b}$ 为预紧条件下导轨副的几何模型图, $D$ 为 滚珠的实际直径, $\delta_{0}$ 为过盈量, $\delta_{0}=2 h_{00}, h_{00}$ 是导 轨副预紧量, $P_{1}$ 是 $\delta_{0}$ 产生的反作用力。预紧条件下 滑块和导轨曲率中心距离 $A B$ 为

$$
\begin{gathered}
A B=r_{\mathrm{a}}+r_{\mathrm{b}}-D=m_{0}+\delta_{0} \\
\delta_{0}=D-D_{0}=2 h_{00}
\end{gathered}
$$




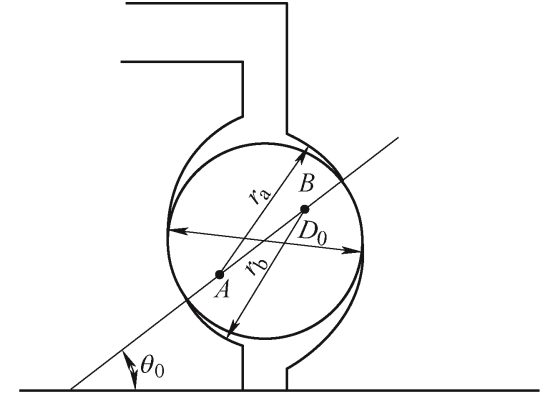

(a) 未预紧时直线导轨的几何模型

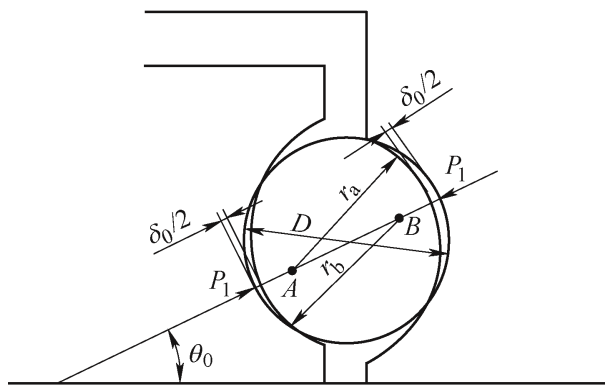

(b) 有预紧时直线导轨的几何模型

图 2 有无预紧时导轨的几何模型

预紧后在垂直载荷 $F_{y}$ 下导轨副的变形如图 $3 \mathrm{a}$ 所示。由图可见, 对导轨副施加垂直载荷 $F_{y}$ 后, 导 轨副产生水平和垂直方向的位移 $\delta_{x} 、 \delta_{y}, Q$ 是滚珠 和导轨接触处的压紧力。

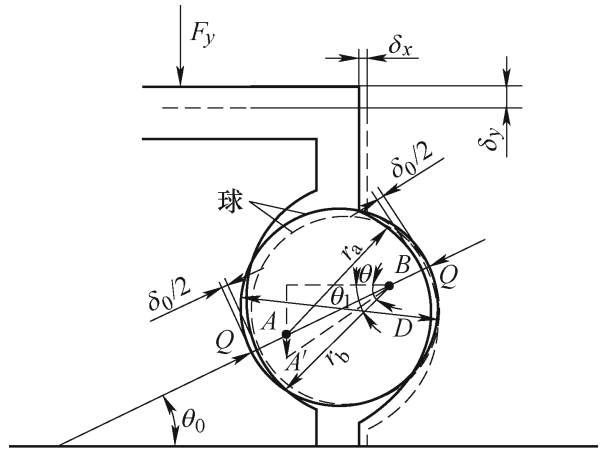

(a) 有预紧并施加垂直载荷时的导轨副变形图

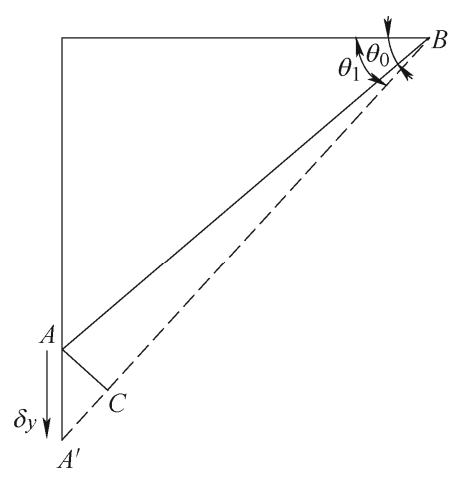

(b) 曲率中心变化模型

图 3 导轨副施加垂直载荷后的结构
变形后滑块的曲率中心为 $A^{\prime}$, 对导轨副施加垂 直载荷 $F_{y}$ 后曲率中心的距离为 $A^{\prime} B$, 导轨副压缩变 形后的接触角为 $\theta_{1}$ 。图 $3 \mathrm{~b}$ 是图 $3 \mathrm{a}$ 中曲率中心变化 图。由于在施加预紧载荷后导轨副的压缩变形过程 中, $\theta_{1}-\theta_{0}$ 很小, 因此作如下假设

$$
A B=C B=m_{0}+\delta_{0}
$$

则 $A^{\prime} B$ 可表示为

$$
A^{\prime} B=A^{\prime} C+C B=m_{0}+\delta_{0}+\delta_{y} \sin \theta_{1}
$$

假设图 $3 \mathrm{~b}$ 中 $A C$ 和 $B C$ 互相垂直, 则可以看出 以下关系

$$
A^{\prime} B \cos \theta_{1}=A B \cos \theta_{0}
$$

将式(6)代入(7)可得

$$
\frac{\cos \theta_{0}}{\cos \theta_{1}}=1+\frac{\delta_{y} \sin \theta_{1}}{m_{0}+\delta_{0}}
$$

接触方向的总变形量 $\delta$ 为滑块与滚珠的变形量 $\delta_{1} 、 \delta_{2}$ 之和

$$
\delta=\delta_{1}+\delta_{2}
$$

$$
\begin{gathered}
\delta_{1}=C_{1} Q^{\frac{2}{3}} \quad \delta_{2}=C_{2} Q^{\frac{2}{3}} \\
C_{1}=\frac{2.79}{10^{4}}\left(\frac{2 K}{\pi \mu}\right)_{1} \sqrt[3]{D_{1} \sum \rho} \\
C_{2}=\frac{2.79}{10^{4}}\left(\frac{2 K}{\pi \mu}\right)_{2} \sqrt[3]{D_{1} \sum \rho}
\end{gathered}
$$

式中, $(2 K / \mu \pi)_{1} 、(2 K / \mu \pi)_{2}$ 表示赫兹系数, 由辅助值 $\cos \tau_{1} 、 \cos \tau_{2}$ 决定，辅助值对应的赫兹系数见文献 [12]。

$$
\cos \tau_{1}=\frac{1}{r_{a} \sum \rho_{1}} \quad \cos \tau_{2}=\frac{1}{r_{b} \sum \rho_{2}}
$$

式中, $\Sigma \rho_{1}$ 和 $\Sigma \rho 2$ 表示曲率和, 可以表示为

$$
\sum \rho_{1}=\frac{4}{D}-\frac{1}{r_{a}} \quad \sum \rho_{2}=\frac{4}{D}-\frac{1}{r_{b}}
$$

在本节中, $2 K / \mu \pi=0.731, C_{1}=C_{2}=2.632 \times 10^{-4}$ 。 导轨副沟槽曲率比 $f$ 又称为导轨副一致性设计参数。 在滚动直线导轨中, 滑块和导轨曲率半径相同, 可 表示为

$$
r_{\mathrm{a}}=r_{\mathrm{b}}=f D
$$

滚珠和导轨接触点处的压缩力 $Q$ 可以表示为

$$
Q=\frac{F_{y}}{i z \sin \theta_{1}}
$$

将式(1)、(9) (11)和(15)代入(8)中可得 


$$
\begin{aligned}
& \frac{\cos \theta_{0}}{\cos \theta_{1}}=1+\frac{\delta}{(2 f-1) D+\delta_{0}}\left(1-\frac{\frac{1}{\delta_{y} \tan \theta_{1}}}{\delta_{x}}\right)= \\
& 1+\frac{\left(C_{1}+C_{2}\right)}{(2 f-1) D+\delta_{0}}\left(\frac{F_{y}}{i z \sin \theta_{1}}\right)\left(1-\frac{\frac{1}{\delta_{y} \tan \theta_{1}}}{\delta_{x}}\right)
\end{aligned}
$$

式中, 由于对导轨施加垂直载荷 $F_{y}$ 后, 水平方向位 移 $\delta_{x}$ 很小且对接触角基本没有影响 ${ }^{[9]}$, 因此式(16) 可简化为

$$
\frac{\cos \theta_{0}}{\cos \theta_{1}}-1=\frac{\left(C_{1}+C_{2}\right)}{(2 f-1) D+\delta_{0}}\left(\frac{F_{y}}{i z \sin \theta_{1}}\right)^{\frac{2}{3}}
$$

由此可通过式(17)求得变形后的接触角 $\theta_{1}$ 。

\section{2 垂直方向位移的计算}

由图 3a 可见, 预紧条件下的导轨副因垂直载荷 $F_{y}$ 发生垂直变形, 曲率中心的距离从 $A B$ 变为 $A^{\prime} B$, 即

$$
A^{\prime} B=r_{\mathrm{a}}+r_{\mathrm{b}}-D_{1}+\delta_{0}
$$

式中, $D_{1}$ 是压缩后的滚珠直径, 接触方向的总变形 量 $\delta$ 等效在滚珠上, 故 $D_{1}$ 可以表示为

$$
D_{1}=D-\delta
$$

因此式(18)可表示为

$$
A^{\prime} B=r_{\mathrm{a}}+r_{\mathrm{b}}-D+\delta+\delta_{0}
$$

将式(3)、(20)代入式(7)中可得

$$
\begin{aligned}
& \left(r_{a}+r_{b}-D+\delta_{0}+\delta\right) \cos \theta_{1}= \\
& \left(r_{a}+r_{b}-D+\delta_{0}\right) \cos \theta_{0}
\end{aligned}
$$

将式(14)代入(21)可得

$$
\delta=\frac{\left[D(2 f-1)+\delta_{0}\right]\left(\cos \theta_{0}-\cos \theta_{1}\right)}{\cos \theta_{1}}
$$

由图 3b 可知

$$
\begin{gathered}
\delta_{y}=A^{\prime} B \sin \theta_{1}-A B \sin \theta_{0} \\
\delta_{y}=A B\left(\sin \theta_{1}-\sin \theta_{0}\right)+\delta \sin \theta_{1}
\end{gathered}
$$

将式(3)、(21)代入(24), 可得 $y$ 方向的变形量为 $\delta_{y}=\left[D(2 f-1)+\delta_{0}\right]\left(\sin \theta_{1}-\sin \theta_{0}\right)+$

$$
\left[\frac{\left(D(2 f-1)+\delta_{0}\right)\left(\cos \theta_{0}-\cos \theta_{1}\right)}{\cos \theta_{1}}\right] \sin \theta_{1}
$$

\section{3 垂直方向刚度系数计算}

在预紧条件下, 对导轨副施加垂直载荷 $F_{y}$ 后, 垂直方向的刚度系数 $K$ 可表示为

$$
K=\frac{F_{y}}{\delta_{y}}
$$

\section{4 刚度计算方法}

求解导轨副刚度系数的步骤如下。

(1)给定垂直载荷 $F_{y}$, 沟槽列数 $i$, 沟槽曲率比 $f$, 参考直径 $D_{0}$, 实际直径 $D$, 单列沟槽有效承载球数 $z$, 过盈量 $\delta_{0}$, 初始接触角 $\theta_{0}$ 。首先确定式(11)中 $C_{1}$ 和 $C_{2}$ 的值, 再根据式(17)计算施加垂直载荷 $F_{y}$ 后导轨副 的接触角 $\theta_{1}$ 。

(2)根据计算得到的 $\theta_{1}$, 计算式(25)中的 $\delta_{y}$ 。

(3) 分别改变预紧量 $h_{00}$ 、曲率比 $f$ 、滚珠直径 $D$ 、 垂直载荷 $F_{y}$ 的参数值。

(4)通过式(26)计算刚度系数 $K$ 。

另外, 在不同的接触副中, 式(22)中接触方向 总变形量 $\delta$ 满足以下关系

$$
\delta=\omega_{\mathrm{E}} \frac{\left[D(2 f-1)+\delta_{0}\right]\left(\cos \theta_{0}-\cos \theta_{1}\right)}{\cos \theta_{1}}
$$

式中, $\omega_{\mathrm{E}}$ 是点接触变形适应系数 ${ }^{[12]}$ 。

$$
\omega_{\mathrm{m}}=\frac{1}{2}\left(\frac{E_{1}}{1-v_{1}^{2}}\right)\left(\frac{1-v_{1}^{2}}{E_{1}}+\frac{1-v_{2}^{2}}{E_{2}}\right)
$$

式中, $v$ 是泊松比, $E_{1} 、 E_{2}$ 分别表示弹性模量。

$$
\omega_{\mathrm{E}}=\omega_{\mathrm{m}}{ }^{2 / 3}
$$

钢 - 钢和钢 $-\mathrm{Si}_{3} \mathrm{~N}_{4}$ 陶瓷两种接触副的材料参数 及变形适应系数如表 1 所示。

表 1 两种接触副的变形适应系数

\begin{tabular}{cc}
\hline 参数 & 内容 \\
\hline 滑块材料 & 钢 \\
弹性模量 $E_{1} /\left(\mathrm{N} / \mathrm{mm}^{2}\right)$ & $2.08 \times 10^{5}$ \\
泊松比 $v_{1}$ & 0.3 \\
滚珠材料 & 钢, $\mathrm{Si}_{3} \mathrm{~N}_{4}$ \\
弹性模量 $E_{2} /\left(\mathrm{N} / \mathrm{mm}^{2}\right)$ & $2.08 \times 10^{5}, 3.2 \times 10^{5}$ \\
弹性模量 $v_{2}$ & $0.30,0.26$ \\
变形适应系数 $\omega_{\mathrm{E}}$ & $1.00,0.83$ \\
\hline
\end{tabular}

\section{3 预紧条件下的弹流润滑模型}

\subsection{Reynolds 方程}

Reynolds 方程如下

$$
\frac{\partial}{\partial x}\left(\frac{\rho h^{3}}{\eta} \frac{\partial p}{\partial x}\right)+\frac{\partial}{\partial y}\left(\frac{\rho h^{3}}{\eta} \frac{\partial p}{\partial y}\right)=12 u \frac{\partial(\rho h)}{\partial x}
$$

式中, $\rho$ 为润滑油的密度, $h$ 为油膜厚度, $\eta$ 为黏度, $p$ 为压力。 
Reynolds 方程的边界条件为

$$
\left\{\begin{array}{l}
p\left(x_{\text {in }}, y\right)=p\left(x_{\text {out }}, y\right)=p\left(x, y_{\text {in }}\right)=p\left(x, y_{\text {out }}\right) \\
p(x, y) \geqslant 0 \quad x_{\text {in }}<x<x_{\text {out }} \quad y_{\text {in }}<y<y_{\text {out }}
\end{array}\right.
$$

式中, $x_{\text {in }}$ 和 $x_{\text {out }}$ 分别表示求解域起止点坐标, $y_{\text {in }}$ 和 $y_{\text {out }}$ 分别表示求解域沿 $y$ 方向的坐标。

\section{2 黏压方程}

Roelands 黏压关系 ${ }^{[13]}$ 可表示为

$$
\eta=\eta_{0} \exp \left\{\left(\ln \eta_{0}+9.67\right)\left[\left(1+5.1 \times 10^{-9} p\right)^{z_{0}}-1\right]\right\}
$$

式中, $\eta_{0}$ 表示环境黏度, $z_{0}$ 与 Barus 黏压系数 $\alpha$ 的 关系为

$$
z_{0}=\alpha /\left[5.1 \times 10^{-9}\left(\ln \eta_{0}+9.67\right)\right]
$$

\section{3 密压方程}

密压关系 ${ }^{[14]}$ 可表示为

$$
\rho=\rho_{0}\left(1+\frac{0.6 \times 10^{-9} p}{1+1.7 \times 10^{-9} p}\right)
$$

式中, $\rho_{0}$ 为润滑油的环境密度。

\section{4 考虑刚度影响的预紧量}

导轨副预紧后, 系统刚度必然对预紧量产生影 响。考虑刚度的影响, 则有

$$
h_{\mathrm{e}}=h_{00}+\frac{1}{K}\left(w-w_{\mathrm{o}}\right)
$$

式中, $h_{00}$ 表示初始预紧量; $w$ 是预紧载荷(由预紧量 $h_{00}$ 决定); $h_{\mathrm{e}}$ 定义为系统弹性预紧量。

\section{5 膜厚方程}

考虑预紧刚度时, 导轨副的油膜厚度方程为 ${ }^{[15]}$

$$
\begin{aligned}
h(x, y)= & -h_{\mathrm{e}}+\frac{x^{2}}{2 R_{x}}+\frac{y^{2}}{2 R_{y}}+ \\
& \frac{2}{\pi E^{\prime}} \iint \frac{p\left(x^{\prime}, y^{\prime}\right) \mathrm{d} x^{\prime} \mathrm{d} y^{\prime}}{\sqrt{\left(x-x^{\prime}\right)^{2}+\left(y-y^{\prime}\right)^{2}}}
\end{aligned}
$$

式中, $R_{x}$ 和 $R_{y}$ 为 $x$ 和 $y$ 两个方向的等效曲率半径, $E^{\prime}$ 为等效弹性模量。

\section{6 油膜承载力方程}

由于导轨副在施加预紧后, 油膜承载力是变化 的, 因此, 在预紧条件下, 不需要载荷平衡方程。 为探究改变工作参数后油膜承载力的变化, 将油膜 承载力 $w_{0}$ 表示为

$$
w_{\mathrm{o}}=\iint p \mathrm{~d} x \mathrm{~d} y
$$

\section{7 接触参数}

根据 Hertz 接触理论, 有关参数计算用到以下 公式

$$
\left\{\begin{array}{l}
R_{x}=\frac{D}{2} \quad \gamma=\frac{R_{y}}{R_{x}} \quad R_{y}=\frac{f D}{2 f-1} \\
R=\frac{R_{x} R_{y}}{R_{y}-R_{x}} \quad b=\left(\frac{6 k_{\mathrm{e}}^{2} F_{2} R w}{E^{\prime} \pi}\right)^{\frac{1}{3}} \\
a=\frac{b}{k_{\mathrm{e}}} \quad p_{\mathrm{H}}=\frac{3 w}{2 \pi a b}
\end{array}\right.
$$

式中, $F_{2}$ 为第二类椭圆积分, $F_{2}$ 由 Marho 回归经验 公式求得

$$
\begin{aligned}
F_{2}= & {\left[1+\left(\frac{\pi}{2}-1\right) \gamma^{-1.0238}\right] \times } \\
& {\left[1+0.0486 \gamma^{-1.3358}(\ln \gamma)^{1.0997}\right] }
\end{aligned}
$$

椭圆比 $k_{\mathrm{e}}$ 计算公式 ${ }^{[16]}$ :

$$
k_{\mathrm{e}}=\left(\frac{R_{y}}{R_{x}}\right)^{0.64}
$$

\section{8 方程的无量纲参数}

方程无量纲化后方可进行数值计算, 给定无量 纲参数为: $P=p / p_{\mathrm{H}}, H=h / h_{0}, h_{0}=a^{2} / R_{x}, \bar{\eta}=\eta / \eta_{0}$, $\bar{\rho}=\rho / \rho_{0}, X=x / a, Y=y / a, U=u \eta_{0} /\left(E^{\prime} R_{x}\right), G=\alpha E^{\prime}$, $W=w /\left(E^{\prime} R_{x}^{2}\right), H_{00}=h_{00} / h_{0}, h_{00}=a^{2} / R_{x}$, 在本节无 量纲参数下, $H_{00}$ 恒等 1 。

\section{4 计算结果及分析}

为验证第 2 部分刚度理论算法的可行性，选用 孙健利等 ${ }^{[17]}$ 的导轨参数进行分析对比。因参考工况 是导轨副未预紧, 为保证工况统一, 令 $\delta_{0}=0$ 。图 4 中, 圆点代表理论计算值, 方点表示文献[17]中的 参考值。由图可见，理论计算值与参考值整体吻合 度较高, 表明了理论算法的可行性。

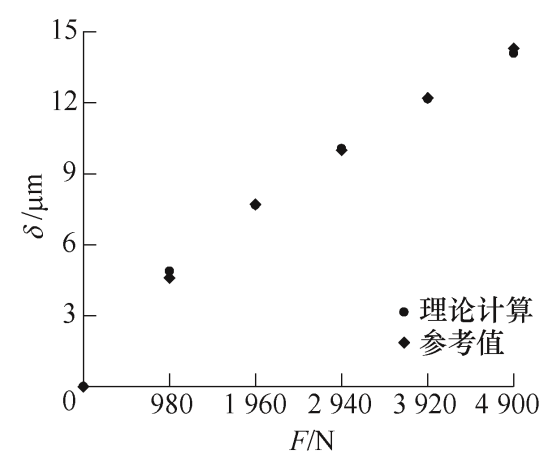

图 4 理论计算与参考值的对比 
图 5 7 是在未受垂直载荷且有预紧条件下, 钢- $\mathrm{Si}_{3} \mathrm{~N}_{4}$ 和钢-钢两种接触副的系统弹性预紧量、预 紧载荷和油膜承载力分别随滑块速度、滚珠直径和 沟槽曲率比的变化。后文提及的 $h_{\mathrm{e}}$ 为系统弹性预紧 量, 简称为预紧量 $h_{\mathrm{e}}$ 。

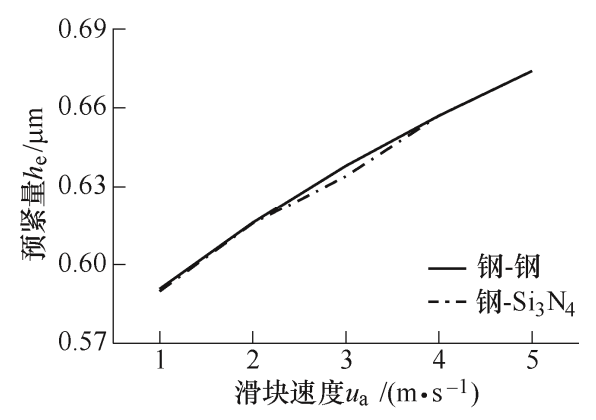

图 5 滑块速度 $u_{\mathrm{a}}$ 对预紧量 $h_{\mathrm{e}}$ 的影响

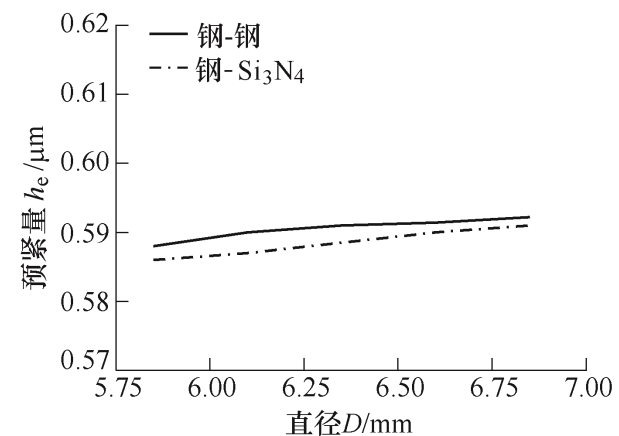

(a) 预紧量 $h_{\mathrm{e}}$ 随滚珠直径 $D$ 的变化
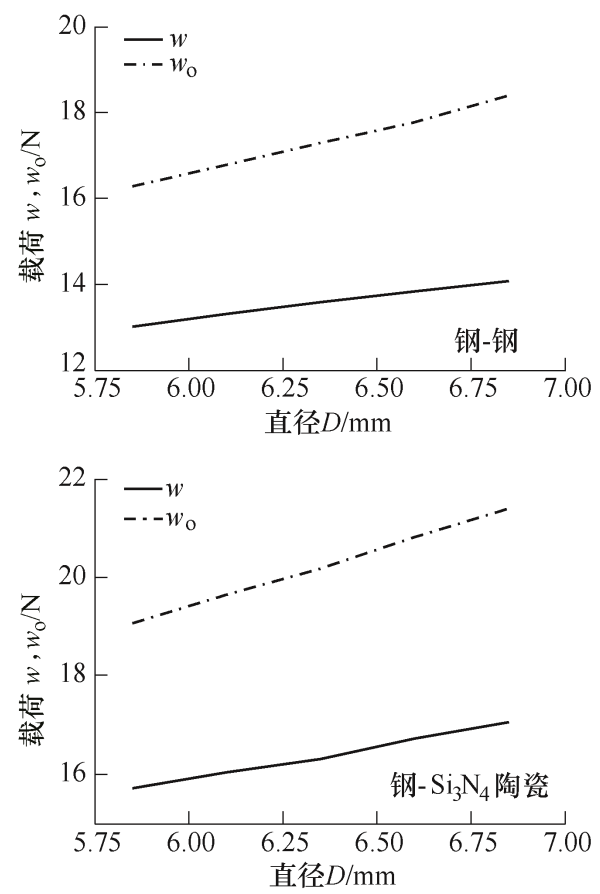

(b) 预紧载荷和油膜承载力随滚珠直径 $D$ 的变化

图 6 滚珠直径 $D$ 对预紧量和承载力的影响

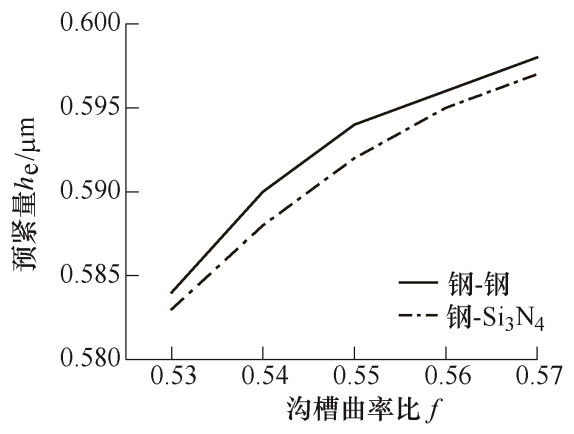

(a) 预紧量 $h_{\mathrm{e}}$ 随沟槽曲率比 $f$ 的变化
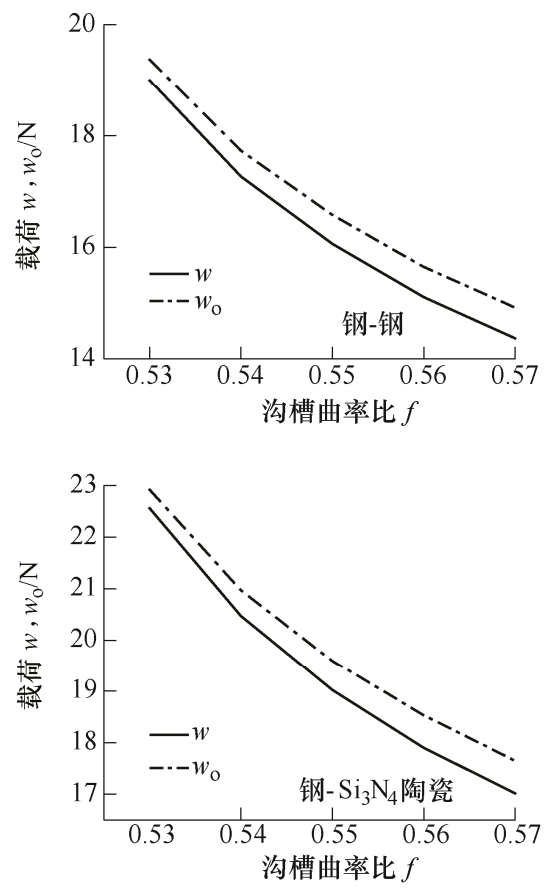

(b) 两种接触副的预紧载荷和油膜 承载力随沟槽曲率比 $f$ 的变化

图 7 沟槽曲率比 $f$ 对预紧量 $h_{\mathrm{e}}$ 和承载力的影响

\section{1 滑块速度 $\boldsymbol{u}_{\mathrm{a}}$ 的影响}

当滚珠直径 $D=6.35 \mathrm{~mm}$, 沟槽曲率比 $f=0.54$, 预紧量 $h_{00}=0.57 \mu \mathrm{m}$ 时, 滑块速度 $u_{\mathrm{a}}$ 对两种接触副 弹性预紧量 $h_{\mathrm{e}}$ 的影响如图 5 所示。由图可知, 受刚 度影响, 滑块速度 $u_{\mathrm{a}}$ 越大, 预紧量 $h_{\mathrm{e}}$ 越大, 但在同 样的滑块速度下, 两种接触副的预紧量 $h_{\mathrm{e}}$ 几乎一致。 其原因是滑块速度 $u_{\mathrm{a}}$ 越大, 油膜承载力越大, 预紧 量 $h_{\mathrm{e}}$ 越大; 与钢对钢接触副相比, 钢对陶瓷接触副 的等效弹性模量较大，使得在同样的速度 $u_{\mathrm{a}}$ 下，刚 度系数越大, 滑块形变越小, 油膜承载力越大, 因 此, 不同接触副对应的系统弹性预紧量相差较小。

\section{2 滚珠直径 $D$ 的影响}

对应预紧量 $h_{00}=0.57 \mu \mathrm{m}$, 沟槽曲率比 $f=$ 0.54 , 滑块速度 $u_{\mathrm{a}}=1.0 \mathrm{~m} / \mathrm{s}$ 时, 图 6 给出了两种 接触副对应的滚珠直径 $D$ 对预紧量和承载力的 影响, 其中, 图 $6 \mathrm{a}$ 为预紧量 $h_{\mathrm{e}}$ 的变化, 图 $6 \mathrm{~b}$ 
为油膜承载力的变化。从图 6 中可以看出, 滚珠 直径 $D$ 越大, 油膜承载力和预紧力越大, 并且两 个力的差别越大。但是预紧量 $h_{\mathrm{e}}$ 随滚珠直径 $D$ 变化不明显, 这是因为在初始预紧量 $h_{00}$ 确定时, 油膜承载力和预紧载荷会随滚珠直径增大而增 大, 这使得滚珠与滑块的接触区域变大, 滚珠的 接触应力变小, 导轨副的变形量小幅度减小, 刚 度系数 $K$ 随滚珠直径 $D$ 变化较小, 因此, 预紧 量 $h_{\mathrm{e}}$ 小幅上升。两种接触副弹性预紧量存在差 别, 是因为与钢-钢接触副相比, 钢-陶瓷接触副 的等效弹性模量较大, 变形较小。

\section{3 沟槽曲率比 $f$ 的影响}

当滚珠直径 $D=6.35 \mathrm{~mm}$, 预紧量 $h_{00}=$ $0.57 \mu \mathrm{m}$, 滑块速度 $u_{\mathrm{a}}=1.0 \mathrm{~m} / \mathrm{s}$ 时, 图 7 给出了沟 槽曲率比 $f$ 对预紧量和承载力的影响, 其中图 $7 \mathrm{a}$ 为 两种接触副的预紧量 $h_{\mathrm{e}}$, 图 7b 分别对应预紧载荷和 油膜承载力。从图中可以看出, 对应钢-钢和钢-陶 瓷接触副, 沟槽曲率比越大, 预紧载荷和油膜承载 力越小, 且两个载荷的差值越大。但是, 沟槽曲率 比越大, 预紧量 $h_{\mathrm{e}}$ 越大, 增大趋势越小。这是因为, 沟槽曲率比越大, 滚珠的预紧力越小, 油膜承载力 越小, 同时两个力的差别越大。对应本小节的 $f$, 刚 度系数取值范围为 $K=285.7 \sim 192.3 \mathrm{~N} / \mu \mathrm{m}$, 刚度系 数的减小加上预紧载荷和油膜承载力差值增大使得 预紧量 $h_{\mathrm{e}}$ 增大。

\section{5 刚度对导轨副润滑性能的影响}

在进行分析之前需要先求得对应工况的刚度系 数。

\section{1 滑块速度的影响}

对钢 $-\mathrm{Si}_{3} \mathrm{~N}_{4}$ 陶瓷接触副, 当滚珠直径 $D=$ $6.35 \mathrm{~mm}$, 沟槽曲率比 $f=0.54$, 初始预紧量 $h_{00}=$ $0.57 \mu \mathrm{m}$ 时, 图 8 为滑块速度对油膜厚度和压力的 影响。当速度变化时, 该工况下刚度系数不变, $K$ $=250 \mathrm{~N} / \mu \mathrm{m}$ 。由图 8 可见, 滑块速度越大, 油膜 厚度和压力越大, 油膜的出口颈缩和第二压力峰 位置均移向出口区。表 2 给出了预紧载荷和油膜 承载力随滑块速度的变化, 由表可知, 滑块速度 不影响预紧载荷, 但影响油膜承载力 $w_{0}$, 大的 滑块速度引起大的油膜承载力 $w_{\mathrm{o}}$ 和预紧量 $h_{\mathrm{e}}$, 进而使得滑块与滚珠的接触面积增大; 但当滑块 速度引起的油膜增厚效应大于弹性预紧量对油 膜的减薄效应时, 速度越大, 油膜厚度和压力 越大。
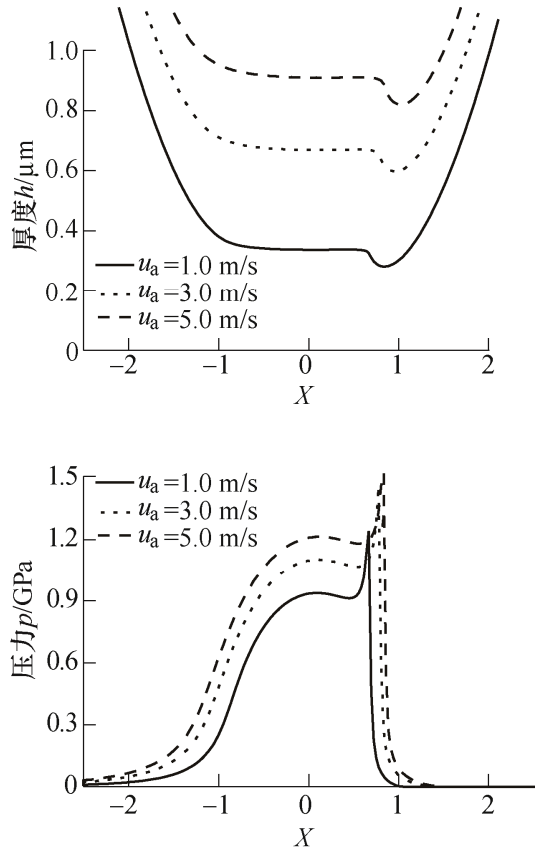

图 8 滑块速度对膜厚和压力的影响

表 2 预紧载荷和油膜承载力随滑块速度的变化

\begin{tabular}{ccc}
\hline 滑块速度 $/(\mathrm{m} / \mathrm{s})$ & 预紧载荷 $w / \mathrm{N}$ & 油膜承载力 $w_{\mathrm{o}} / \mathrm{N}$ \\
\hline 1.0 & 16.32 & 20.41 \\
3.0 & 16.32 & 32.54 \\
5.0 & 16.32 & 41.88 \\
\hline
\end{tabular}

\section{2 沟槽曲率比的影响}

当滚珠直径 $D=6.35 \mathrm{~mm}$, 滑块速度 $u_{\mathrm{a}}=5.0$ $\mathrm{m} / \mathrm{s}$, 初始预紧量 $h_{00}=0.57 \mu \mathrm{m}$ 时, 油膜厚度和压力 随沟槽曲率比 $f$ 的变化见图 9 。当沟
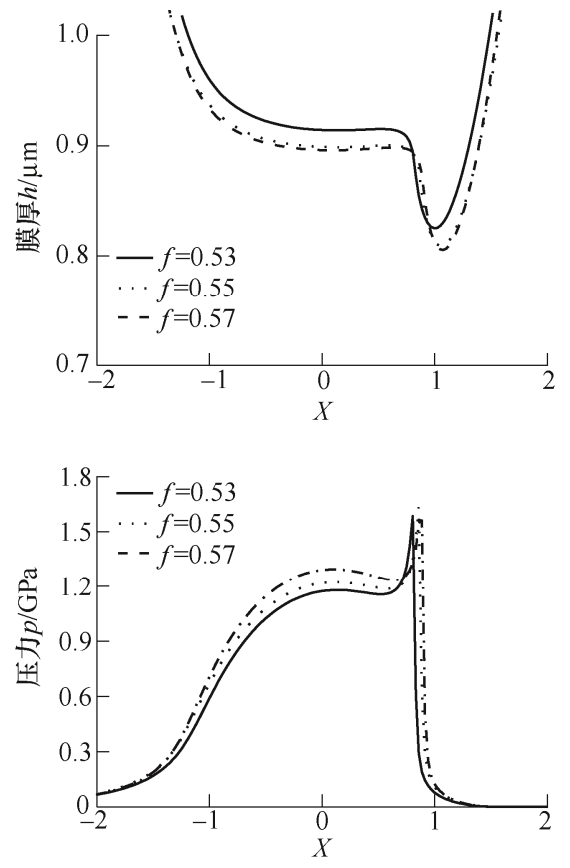

图 9 沟槽曲率比对膜厚和压力的影响 
槽曲率比为 $f=0.53,0.55,0.57$, 对应的刚度系数 分别为 $K=285.7 \mathrm{~N} / \mu \mathrm{m}, 208.3 \mathrm{~N} / \mu \mathrm{m}, 192.3 \mathrm{~N} / \mu \mathrm{m}$ 。 从图 9 中可以看出, 沟槽曲率比越大, 油膜厚度越 小, 油膜压力越大。这是因为大的沟槽曲率比会降 低滚珠预紧力, 减小油膜承载力, 并增大二个力之 间的差异。同时, 刚度系数随曲率比的增大而减小, 使得预紧量 $h_{\mathrm{e}}$ 增大, 膜厚减小, 油膜压力增大。

\section{3 滚珠直径的影响}

当滑块速度 $u_{\mathrm{a}}=5.0 \mathrm{~m} / \mathrm{s}$, 沟槽曲率比 $f=0.54$, 初始预紧量 $h_{00}=0.57 \mu \mathrm{m}$ 时, 图 10 给出了油膜厚度 和压力随滚珠直径 $D$ 的变化。对应滚珠直径 $D=$ $5.85 \mathrm{~mm}, 6.35 \mathrm{~mm}, 6.85 \mathrm{~mm}$ 时的刚度系数 $K=$ $238.1 \mathrm{~N} / \mu \mathrm{m}, 250 \mathrm{~N} / \mu \mathrm{m}, 256.4 \mathrm{~N} / \mu \mathrm{m}$ 。从图中可以 看出, 滚珠直径越大, 油膜厚度越大, 油膜压力越 小。这是因为, 虽然预紧量几乎不受受滚珠直径的 影响, 但当增加滑块速度时, 油膜厚度显著增加。
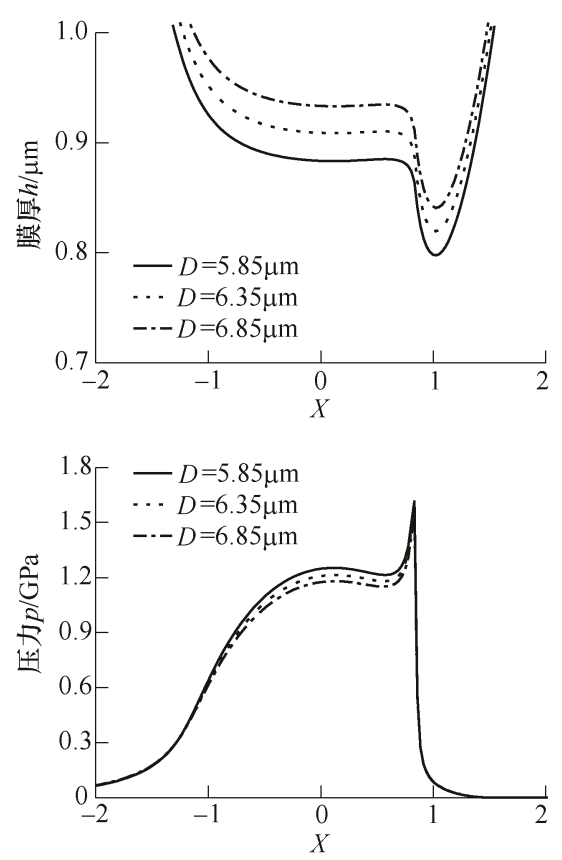

图 10 滚珠直径对膜厚和压力的影响

\section{6 结论}

(1) 系统弹性预紧量随滚珠直径和沟槽曲率比 变化不大, 而随滑块速度变化显著。

(2) 导轨润滑性能随刚度的变化而变化, 滑块 速度越大, 预紧量越大, 油膜厚度和压力越大。

(3) 随曲率比越大, 刚度系数越小, 预紧量越 大, 使得油膜厚度变薄, 而压力升高。

(4)滚珠直径的增大对系统弹性预紧量影响不 大, 速度较高时, 大的滚珠直径引起较大的油膜厚 度和较小的油膜压力。

\section{参 考 文 献}

[1] OHTA H, TANAKA K. Vertical stiffnesses of preloaded linear guideway type ball bearings incorporating the flexibility of the carriage and rail[J]. ASME Journal of Tribology, 2010, 132: 1-9.

[2] LI X P, LIU Y L, LIU J N. Theory and experimental research on static stiffness of linear rolling guide[C]// 2012 IEEE International Conference on Computer Science and Automation Engineering, 2012，2: 85-88.

[3] 张巍, 王民, 孙乐乐. 考虑预紧力和接触角变化的直线 导轨副刚度建模与分析 [J]. 北京工业大学学报, 2018, 44(1): 56-63.

ZHANG Wei, WANG Min, SUN Lele. Stiffness modeling and analysis of linear guide pair considering pretension force and contact angle variation[J]. Journal of Beijing University of Technology, 2018, 44(1): 56-63.

[4] TONG V C, KHIM G, HONG S W. Construction and validation of a theoretical model of the stiffness matrix of a linear ball guide with consideration of carriage flexibility[J]. Mechanism and Machine Theory, 2019, 140: $123-143$.

[5] RAHMANI M , BLEICHER F. Experimental and analytical investigations on normal and angular stiffness of linear guides in manufacturing systems[J]. The 48th CIRP Conference on Manufacturing Systems, 2016, 41: 795-800.

[6] 田红亮, 余媛, 杜义贤. 重载下滚动直线导轨副的接触 特性 [J]. 西安交通大学学报, 2018, 52(9): 1-9.

TIAN Hongliang, YU Yuan, DU Yixian. Contact characteristics of rolling linear guide pair under heavy load [J]. Journal of Xi'an Jiaotong University, 2018, 52(9): 1-9.

[7] DUNAJ P, BERCZYŃSKI S, PAWEŁKO P. Static condensation in modeling roller guides with preload[J]. Archives of Civil and Mechanical Engineering, 2019, 19(4), 1072-1082.

[8] HUNG J P, LIN C Y, LUO T L. Fault detection of linear guide preload of a positioning stage with vibration-acoustic analysis[J]. Journal of Failure Analysis and Prevention, 2011, 11(6): 684-692.

[9] HUNG J P, LAI Y L, CHEN Y R. Dynamic characteristics of a gantry milling machine under the influence of linear guide preload[J]. Applied Mechanics and Materials, 2013, 404: 194-199. 
[10] CHING Y L, JUI P H, TZUO L L. Effect of preload of linear guides on dynamic characteristics of a vertical column spindle system[J]. International Journal of Machine Tools and Manufacture, 2010, 8: 741-746.

[11] SHAW D, SU W L. Stiffness analysis of linear guideways without preload[J]. Journal of Mechanics，2013，29(2): 281-286.

[12] BRÄNDLEIN J. Ball and roller bearings[M]. Theory, Design, and Application, 1999, 102-108.

[13] ROELANDS C J A. Correlation aspects of viscosity-temperature-pressure relationship of lubricating oils[D]. Delft, Netherlands: technischehogeschool delft, 1966.

[14] DOWSON D, HIGGINSON G R. Elasto-hydrodynamic lubrication[M]. Pergamon Press, 1977.

[15] 刘广媛. 限制空间弹性流体动力润滑的特性研究[D]. 青岛: 青岛理工大学, 2013 .

LIU Guangyuan. Study on the characteristics of space-constrained elastohydrodynamic lubrication[D]. Qingdao: Qingdao University of Technology, 2013.
[16] 张朝辉, 孙健利. 滚动直线导轨的弹流润滑分析 [J]. 机 械设计，1997(9): 27-29.

ZHANGZhaohui , SUN Jianli. Analysis of Elastohydrodynamic Lubrication of Rolling Linear Guides[J]. Mechanical Design, 1997(9): 27-29.

[17] 孙健利. 直线滚动导轨机构承受垂直载荷时的刚度计 算[J]. 华中理工大学学报, 1988, 15(5): 35-40.

SUN Jianli. Stiffness calculation of linear rolling guide mechanism under vertical load[J]. Journal of Huazhong University of Science and Technology, 1988, 15(5): $35-40$.

[18] 李栋. 滚动直线导轨的弹流润滑数值分析[D]. 青岛: 青 岛理工大学, 2019 .

LI Dong. Numerical analysis of elastohydrodynamic lubrication of rolling linear guide[D].Qingdao: Qingdao University of Technology, 2019.

作者简介: 李栋, 男, 1993 年出生。主要研究方向为润滑理论。 E-mail: 15610471707@163.com 刘晓玲(通信作者): 女, 1972 年出生, 博士, 教授, 博士研究生导师。 主要研究方向为润滑理论。

E-mail: liu_xiaoling06@126.com 